\title{
Il patrimonio dell'elettricità: una risorsa culturale da valorizzare
}

DOSSIER PATRIMONIO DELL'ELETTRICITÀ: CITTÀ E PAESAGGI ELETTRICI

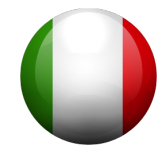
Manuela Mattone

Architetto, P.h.D., Professore associato, Politecnico di Torino. Torino [Piemonte] Italia $<$ manuela.mattone@polito.it>.

\section{Riassunto}

I paesaggi e le architetture elettriche rappresentano una significativa testimonianza della storia che ha visto protagonisti numerosi paesi tra il XIX e il XX secolo. Si tratta di un patrimonio tuttora solo parzialmente indagato, rispetto al quale occorre farsi promotori di azioni volte a favorirne la conoscenza, la conservazione e non ultime la valorizzazione e fruizione da parte di un pubblico sempre più ampio e non necessariamente specializzato. I manufatti connessi alla produzione dell'energia idroelettrica e le tracce delle opere infrastrutturali resesi necessarie al momento della loro costruzione rappresentano una vera e propria risorsa culturale che, qualora integrata alle altre risorse presenti nei territori montani, potrebbe acquisire maggiore visibilità e leggibilità contribuendo a rendere questi ambiti appetibili e ricercati non solo per gli aspetti di carattere naturalistico e paesaggistico, ma anche storico-culturale, contribuendo alla loro riattivazione.

\section{Parole chiave}

Patrimonio idroelettrico. Risorse. Energy tourism. Valorizzazione. Fruizione.

\section{Electrical heritage: a cultural asset to be enhanced}

\begin{abstract}
Landscapes and electrical architectures represent a significant evidence of the history of many countries between the 19th and 20th centuries. It is a heritage that, till now, has only been partially investigated and whose knowledge, conservation, and ultimately enjoyment of an ever wider and not necessarily specialized public should be promoted. The artifacts connected with the production of hydroelectric power and the traces of the infrastructural works necessary for their construction represent a real cultural resource that, if integrated with other resources characterizing the mountainous areas, could gain greater visibility and legibility. They could make these areas more interesting and attractive not only for their natural and landscape assets, but also for the historical-cultural ones, contributing to the re-activation of these territories.
\end{abstract}

\section{Keywords}

Electrical heritage. Assets. Energy tourism. Enhancement. Enjoyment. 


\section{Il patrimonio dell'elettricità}

Gli ultimi decenni dell'Ottocento e i primi del Novecento si caratterizzano, tanto in Italia quanto all'estero, per il verificarsi di eventi destinati a incidere in modo significativo sul settore della produzione e dei trasporti, così come sul paesaggio e sulla società. L'avvio della produzione dell'energia elettrica e la progressiva elettrificazione del territorio hanno infatti determinato quella che è stata definita la "seconda rivoluzione industriale", in relazione ai profondi cambiamenti che da essi ne sono scaturiti tanto a livello produttivo e infrastrutturale quanto a livello sociale.

Energia elettrica non significa [infatti] solo un nuovo genere di illuminazione, ossia una lenta ma radicale trasformazione della percezione del mondo notturno, ma significa anche e soprattutto una rivoluzione ${ }^{1}$ dei trasporti urbani, della produzione industriale e, successivamente, della vita domestica (Castellano, 1991, p. 120).

Il tema dell'elettricità, e in particolare dell'idroelettricità, ha visto convergere nel corso degli ultimi decenni l'interesse di un numero crescente di studiosi impegnati ad approfondire e ad analizzare i diversi aspetti in relazione ai quali tale tema può essere declinato ${ }^{2}$. Per quanto attiene in particolare le tematiche più strettamente connesse al settore dell'architettura, a partire dalla fine degli anni novanta del secolo scorso sono stati avviati studi che hanno visto focalizzare l'attenzione principalmente sulle dighe e sulle centrali che, sovente opere d'autore, rappresentano vere e proprie "emergenze architettoniche". Queste, realizzate per scopi produttivi venivano altresì concepite con l'intento di promuovere e dare lustro e maggiore visibilità alle società produttrici di energia elettrica che tali opere commissionavano. Come sottolineato da Ornella Selvafolta, si trattava infatti di conciliare

la logica dello sfruttamento con le motivazioni simboliche e dell'auto rappresentazione (Selvafolta, 1998, p. 46).

individuando un compromesso tra "l'artificio" e la natura. Ad architetti quale Gaetano Moretti, Piero Portaluppi, Giancarlo Maroni, Giovanni Muzio, Eugenio Mollino e Giò Ponti viene dunque affidato il compito di progettare centrali idroelettriche che, connotate da una particolare linea estetica (Vismara, 1925) e una ricerca di qualità, avrebbero assunto il ruolo di monumenti duraturi (ibidem) tanto da divenire vero e proprio motivo di vanto per l'intera Nazione. Si pensi, ad esempio alla centrale Benigno Crespi a Trezzo d'Adda, progettata da Gaetano Moretti, piuttosto che al sistema di centrali realizzate da Piero Portaluppi nella Val d'Ossola - assimilabili a "gemme incastonate" nelle montagne - o ancora alla centrale di Ponale che, disegnata da Giancarlo Maroni, si adagia lungo le sponde del lago di Garda fondendosi con il paesaggio circostante.

Il sistema dell'idroelettricità si compone tuttavia di numerosi altri elementi sino ad oggi solo in parte studiati e analizzati. Accanto alle "emergenze" architettoniche e ingegneristiche, quali centrali e dighe, esistono numerose altre opere necessarie sia al funzionamento degli impianti idroelettrici, sia alla loro costruzione. Si tratta, nel primo caso, di canalizzazioni coperte o scoperte, condotte forzate, camere di carico, ecc., nel secondo caso, di ferrovie decauville, basi delle teleferiche e delle gru, impianti di frantumazione, baracche destinate a ospitare gli operai, ecc. Le prime, sottoposte a

\footnotetext{
${ }^{1}$ Gli esponenti del Movimento futurista non mancano di celebrare nei loro scritti e nelle loro opera la forza dirompente dell'elettrificazione. Basti pensare ai quadri di Umberto Boccioni, Le forze di una strada (1911) e di Giacomo Balla, Lampada studio della luce (1914) o a quanto scritto sia nel Manifesto del futurismo: noi [...] canteremo il vibrante fervore notturno degli arsenali e dei cantieri incendiati da violente lune elettriche (Marinetti, 1909), sia da Boccioni: infinitamente sublime lo sconvolgere fa l'uomo sotto la spinta della ricerca e della creazione, l'aprire strade, colmare laghi, sommergere isole, lanciare dighe, livellare, squarciare, forare, sfondare, innalzare, per questa divina inquietudine che spara nel futuro (Boccioni, 1914).

${ }^{2}$ Come sottolinea Miguel Ángel Álvarez Areces: la compleja variedad de problemas presentes en el agua como patrimonio, y del patrimonio del agua, concita y promueve una convegencia de esfuerzos de profesionales provenientes de diferentes ámbitos: historiadores, ingenieros, arquitectos, geógrafos, antropólogos, ecólogos, urbanistas, economistas, sociólogos, y, por supuesto, el ciudadano que tiene interiorizado y asumido que el agua es vital para su vida y la de los suyos, para la comunidad en la que está inserto, y que la resolución de los trastornos en su disfrute es competencia global del planeta en su conjunto (Álvarez Areces, 2007, p. 10).
} 
continui interventi di manutenzione, segnano oggi come in passato i territori montani e, benché al momento della loro realizzazione avessero suscitato notevoli polemiche per il loro forte impatto sul territorio ${ }^{3}$, oggi non sono pressoché più percepite come elementi artificiali, poiché totalmente inglobate nel paesaggio. Le seconde viceversa, venute meno le ragioni e le esigenze per cui erano state realizzate, sono state abbandonate al loro destino e si stagliano nel paesaggio come lacerti privati del loro significato. Questi reperti rappresentano segni del progresso, della rivoluzione e dello sviluppo industriali, ma anche segni delle sofferenze e delle difficoltà che, affrontate e superate dalle generazioni che ci hanno preceduto, hanno reso possibile le attuali condizioni di vita (Di Battista, 2011).

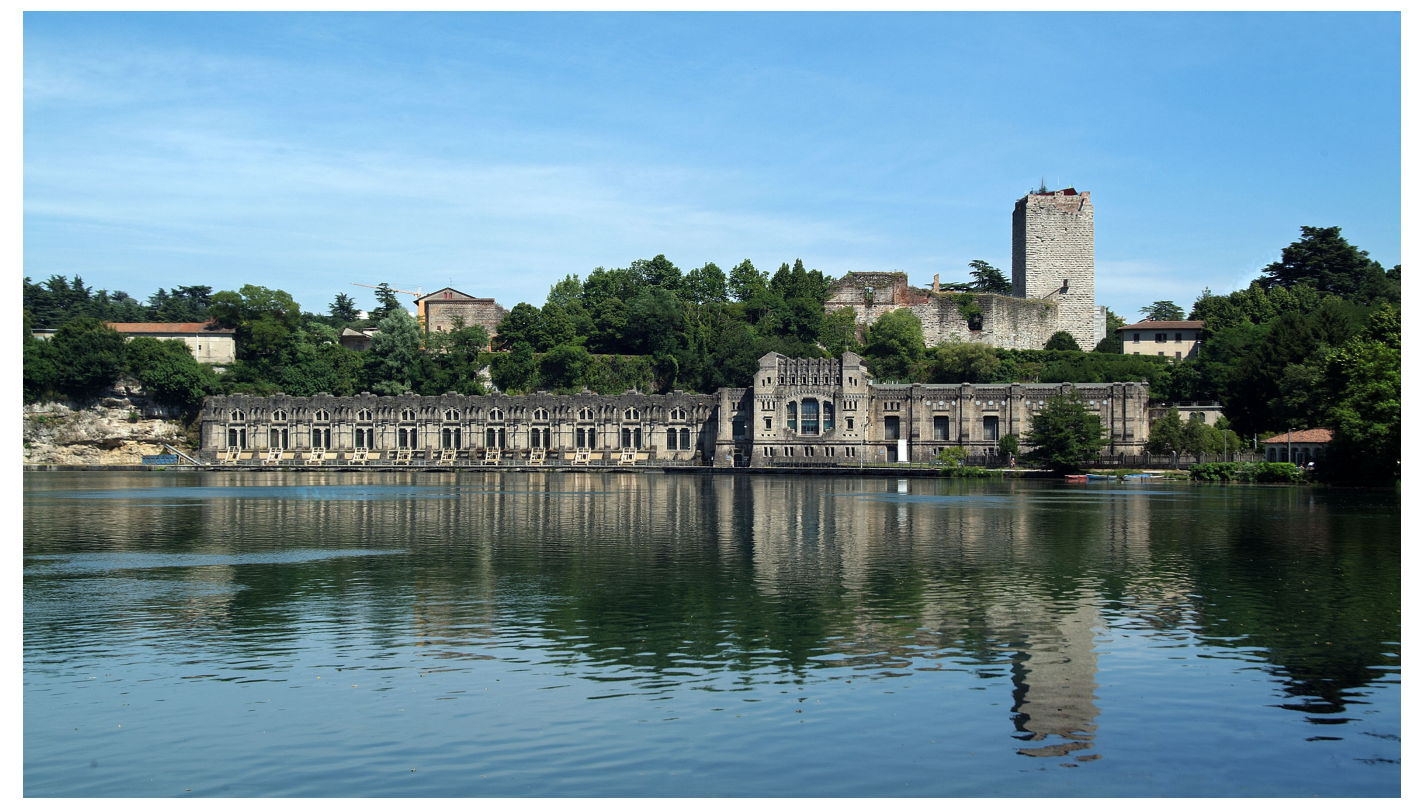

Figura 1. Centrale Benigno Crespi, Trezzo d'Adda (Milano, Italia). Prospetto principale della centrale e retrostante castello medievale (foto Studio Publica).

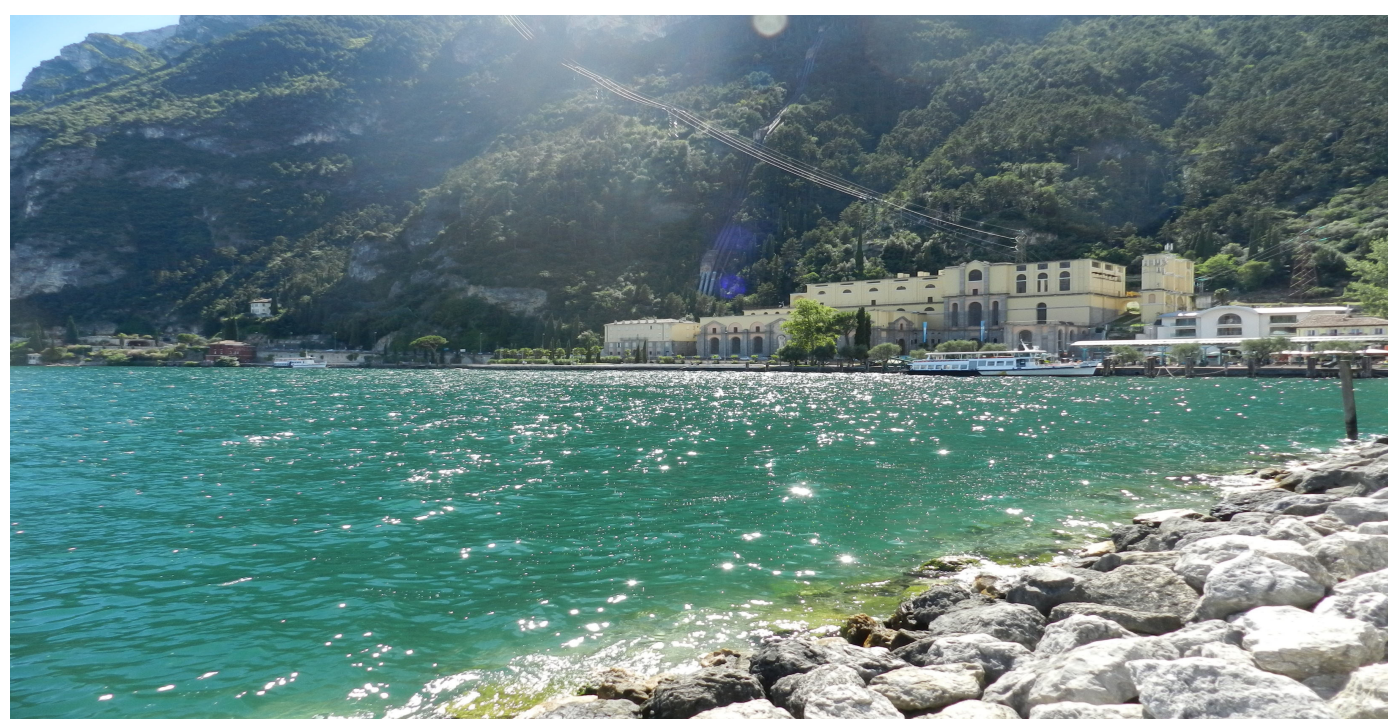

Figura 2. Centrale del Ponale, Riva del Garda (Trento, Italia). Prospetto principale della centrale (foto Manuela Mattone).

\footnotetext{
${ }^{3}$ La realizzazione degli impianti idroelettrici ha sicuramente avuto notevoli ripercussioni sul paesaggio e sulla natura che sono stati sottoposti a modificazioni irreversibili. Al momento della loro costruzione queste non hanno mancato di suscitare dubbi, reazioni, avversità da parte degli esponenti della cultura e della politica. Nel 1923 Luigi Parpagliolo pubblica il volume La difesa delle bellezze naturali d'Italia nel quale sottolinea gli effetti negativi imputabili alla realizzazione di tali impianti, ponendo tuttavia in evidenza come risultasse assolutamente necessario cercare di individuare un giusto compromesso tra il progresso - e tutto ciò che esso avrebbe implicato - e la salvaguardia del patrimonio naturalistico (e architettonico) presente nei territori montani (Mattone, 2017).
} 


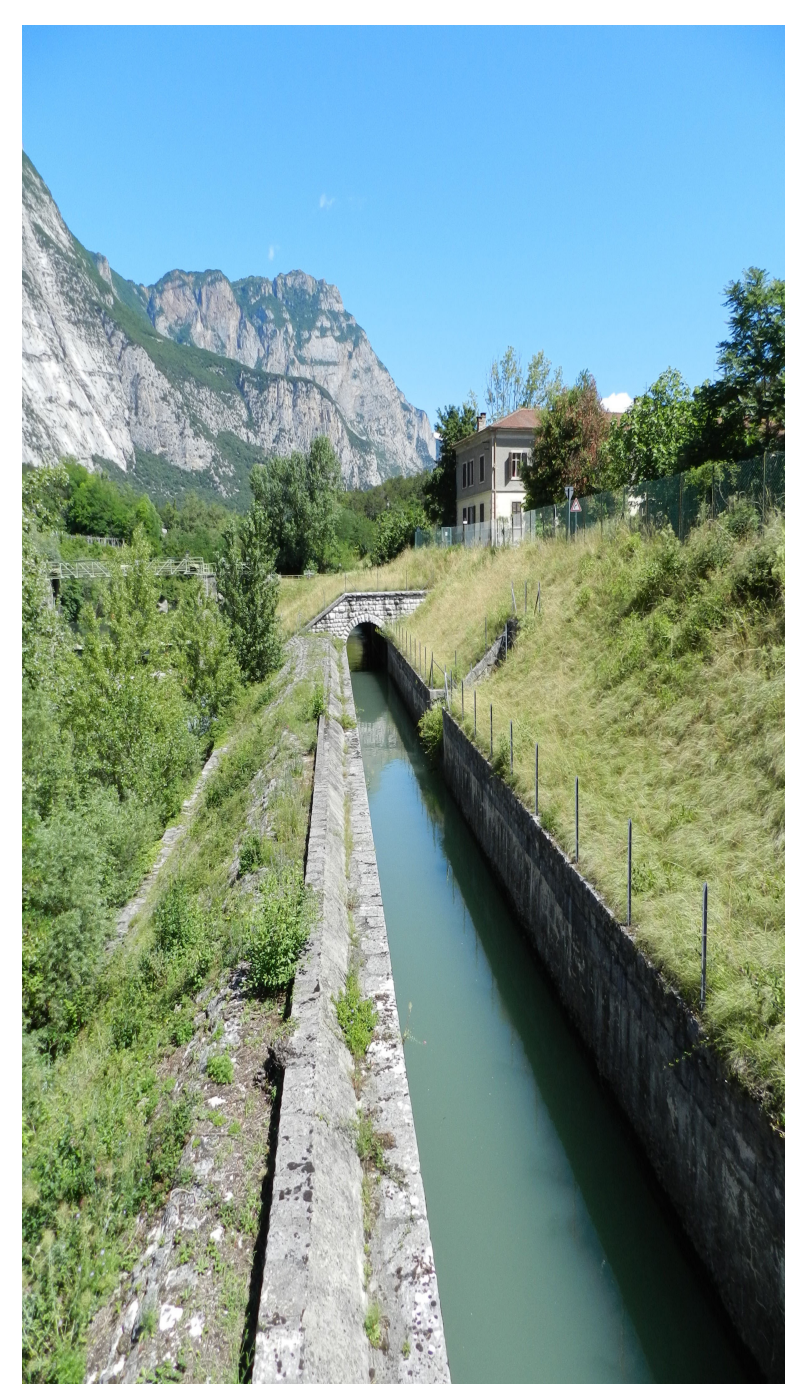

Figura 3. Patrimonio dell'idroelettricità: canalizzazione scoperta in prossimità della centrale di Fies (Trento, Italia) (foto Manuela Mattone).

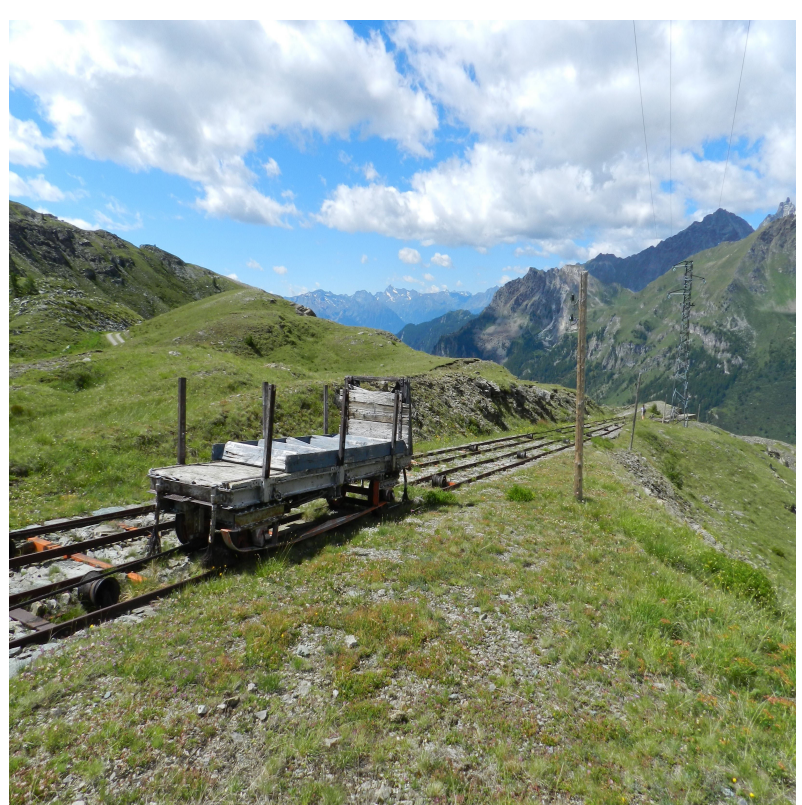

Figura 4. Valtournenche (Val d'Aosta, Italia). Resti della strada ferrata utilizzata per il trasporto dei materiali (foto Manuela Mattone).

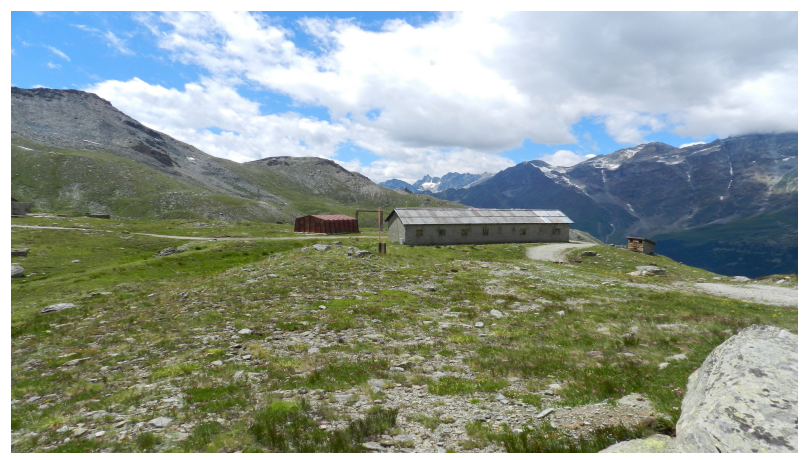

Figura 5. Valtournenche (Val d'Aosta, Italia). Stazione di arrivo della strada ferrata e baracche degli operai in prossimità della diga del Goillet (foto Manuela Mattone).

L'evoluzione di cui è stato protagonista il concetto di patrimonio a partire dalla seconda metà del $\mathrm{XX}$ secolo ne ha determinato una progressiva estensione tipologica, cronologica e geografica ${ }^{4} \mathrm{e}$ ha visto di conseguenza l'incremento e l'ampliamento delle categorie di beni individuabili come testimonianze materiali [e immateriali] aventi valore di civiltà̄ ${ }^{5}$, in quanto tali, da preservare e da trasmettere ai posteri. Secondo quanto sancito in Italia dal Codice dei Beni culturali e del paesaggio, il patrimonio culturale è costituito dai beni culturali e dai beni paesaggistici (D. Lgs. 42/2004 e s.m.i., art. 2, comma 1) che comprendono tanto

le cose immobili e mobili [...] che presentano interesse artistico, storico, archeologico, etnoantropologico [quanto] gli immobili e le aree [...] costituenti espressione di valori storici, culturali, naturali, morfologici ed estetici del territorio (D. Lgs. 42/2004 e s.m.i., art. 2, commi 2 e 3 ).

\footnotetext{
${ }^{4} \mathrm{Si}$ vedano a tal proposito i numerosi documenti, esito di un ampio dibattito disciplinare, redatti a partire dagli anni sessanta ad oggi. Cfr. Choay, F. (1995) L'allegoria del patrimonio. Roma: Officina Edizioni; Vecco, M. (2007). L'evoluzione del concetto di bene culturale. Milano: FrancoAngeli; Vecco, M. (2010). A definition of cultural heritage: from the tangible to the untangible. In Journal of Cultural Heritage, 11: 321-324.
}

5 La definizione è riportata nel documento elaborato dalla Commissione Franceschini e pubblicato nel 1967 con il titolo Per la salvezza dei beni culturali in Italia, Roma: Casa editrice Colombo. 
I beni materiali e immateriali connessi alla produzione dell'elettricità e, nel caso specifico esaminato, dell'idroelettricità costituiscono a tutti gli effetti beni culturali e paesaggistici che, come sottolineato dallo stesso Codice, dovrebbero essere tutelati, per "preservare la memoria della comunità nazionale e del suo territorio" (art. 1, comma2), e valorizzati promuovendone la conoscenza e assicurandone le migliori condizioni di utilizzazione e pubblica fruizione (art. 6, comma 1). Tali beni risultano ad oggi solo in parte conservati e avvalorati. Mentre le opere architettoniche e infrastrutturali inserite nel circuito produttivo sono state oggetto di continui interventi manutentivi atti a garantirne il perfetto funzionamento e l'efficienza nel tempo, tutte quelle opere che, viceversa, hanno perso il loro status produttivo sono state progressivamente abbandonate e hanno ormai, in molti casi, assunto lo status di rudere. Si tratta tuttavia in entrambi i casi di beni che, rientrando in quello che può essere identificato come patrimonio dell'elettricità, si connotano non solo (e, in taluni casi, non più) come risorse produttive, ma anche come risorse culturali oggi solo parzialmente riconosciute come tali.

Abbandonata la ricerca selettiva delle sole "emergenze" si ritiene ora opportuno, alla luce della maggiore estensione del concetto di bene culturale, valutare con più attenzione le molteplici tracce che nel tempo si sono sedimentate sul territorio. Queste tracce presentano valori testimoniali e sociali a oggi non sempre decodificati o facilmente decodificabili. Esse

conservano la memoria della costruzione di quelle opere: le storie dei tecnici, degli operai, l'eco del loro successo e del loro sacrificio sono ancora lì. Quelle tracce ingombranti, dimenticate, private del loro significato di testimonianza, nascondono il segreto dell'attuazione delle grandi opere (Pavia, 1998, p. 339).

Esse sono simboli di identità locale e al contempo testimoniano la storia di un Paese e del suo progresso. Il riconoscimento e la fruizione di tali beni potrebbero contribuire in modo significativo a una maggiore diffusione e a una presa di coscienza da parte di un più ampio pubblico relativamente alle modalità di produzione dell'energia elettrica, ma anche relativamente alla storia dell'elettrificazione, agli sforzi e all'impegno che essa ha richiesto, all'impatto che ha esercitato sul territorio e sugli uomini che sono stati protagonisti di questa impresa.

Occorre inoltre ricordare che la realizzazione degli impianti idroelettrici aveva di fatto determinato, all'inizio del secolo scorso, l'instaurarsi di un nuovo rapporto con i territori montani. Divenuti più facilmente accessibili, e dunque fruibili, grazie alle opere infrastrutturali (strade ferrate e carrabili) realizzate in concomitanza con l'installazione di detti impianti, questi iniziarono ad essere guardati con occhi diversi e valutati ravvisando in essi sia un valore culturale, sia un valore economico legato al progressivo sviluppo del turismo di cui divennero protagonisti. In diversi casi infatti lo sfruttamento elettrico delle valli non avvenne a discapito della loro vocazione turistica, ma, al contrario, ne favorì la conoscenza e la fruizione da parte di coloro che, sfruttando le nuove infrastrutture, sceglievano di trascorrere il proprio tempo libero in montagna ${ }^{6}$ (Bettini, 1991). Oggi alle risorse di carattere naturalistico si sommano quelle di carattere culturale; la dimensione turistica di questi luoghi non è solo affiancata dalla dimensione elettrica, ma da questa stessa incrementata. Pertanto, gli impianti idroelettrici, se opportunamente integrati con le altre risorse presenti nei territori montani, potrebbero rendere questi stessi ambiti più appetibili e ricercati non solo per gli aspetti di carattere naturalistico e paesaggistico, ma anche storico-culturale, offrendo nuove opportunità di svago e accrescimento culturale. Questo potrebbe altresì costituire incentivo a una graduale riscoperta di questi luoghi da parte delle comunità e con essa una auspicabile inversione dell'attuale tendenza che li ha visti, nel corso degli ultimi decenni, protagonisti di fenomeni di ritrazione demografica con conseguente spopolamento delle vallate.

\section{Valorizzazione di una risorsa culturale: esperienze a confronto}

Nel corso degli ultimi anni si è di fatto assistito a una progressiva integrazione tra tematiche energetiche e turismo. Il manifestarsi di un interesse in costante crescita nei confronti delle diverse

\footnotetext{
${ }^{6}$ Scrive a tal proposito Beatrice Archesso trattando della Cascata del Toce in val Formazza (Piemonte, Italia): fino agli anni 20 il maestoso salto d'acqua si raggiungeva solo a piedi, con un trekking nemmeno troppo confortevole. Poi arrivò la strada carrozzabile negli anni 30, con un secondo fine: la logistica nella prospettiva dell'energia idroelettrica. [...] La Cascata del Toce formazzina quale polo d'attrazione turistica fu una visione avveniristica e continua oggi con bed\&breakfast e piccole strutture ricettive che affiancano le proposte invernali - sci alpinismo e fondo - ed estive con il grande escursionismo (Archesso, 2016).
} 
fonti di produzione di energia elettrica ha infatti, tra le altre cose, determinato lo sviluppo di quello che viene definito "energy tourism". Si tratta di una particolare categoria di turismo industriale che,

como vertiente del turismo cultural, connecta la geografiá del turismo con los itinerarios a las huellas y vestigios de la industria y también a su patrimonio vivo: factoriás, centrales eléctricas, minas, puentes, canales, altos hornos, imprentas, cerámicas y cementeras, todo un conjunto de elementos o monumentos de la industria que describen sendas cargadas de historia y simbolismo (Álvarez Areces, 2017, p. 133),

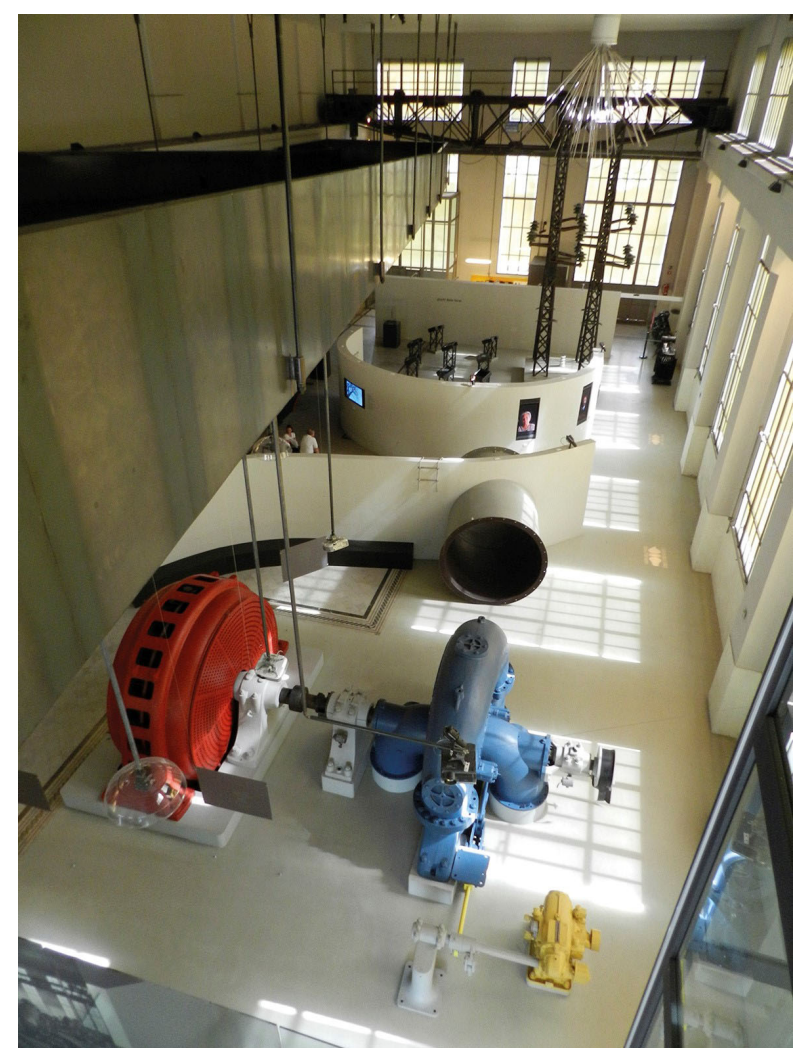

Figura 6. Museo dell'energia idroelettrica. Cedegolo (Brescia, Italia). Particolare del percorso espositivo (foto Manuela Mattone).

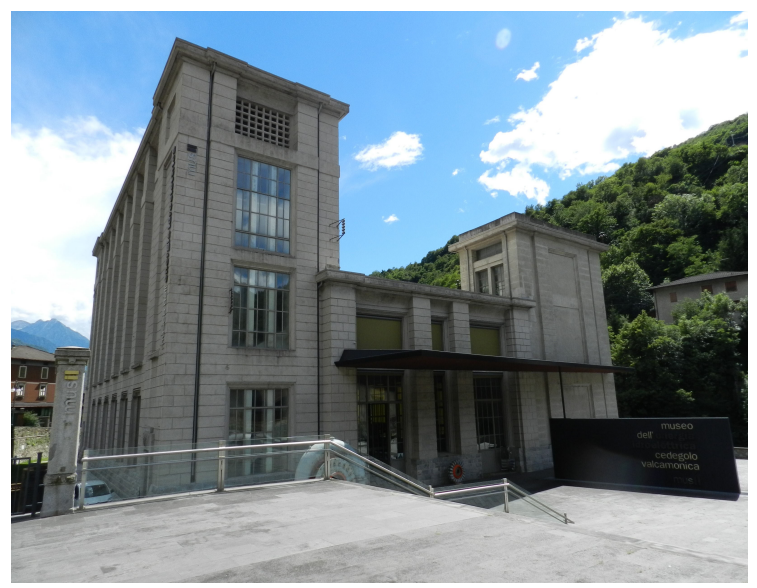

Figura 7. Museo dell'energia idroelettrica. Cedegolo (Brescia, Italia) (foto Manuela Mattone).

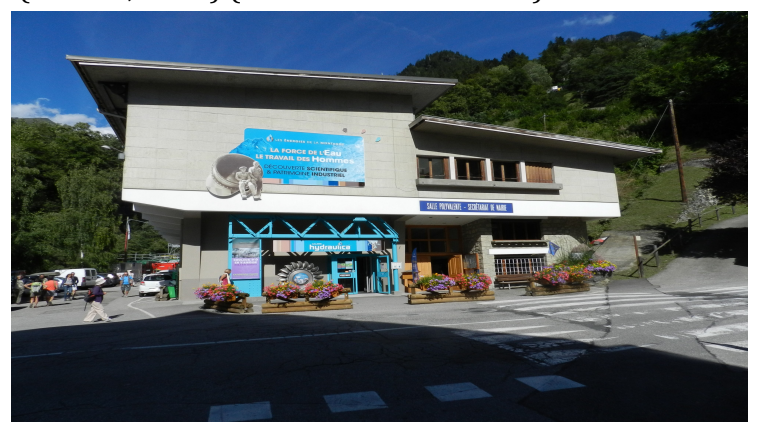

Figura 8. La Galerie Hydraulyca a Le Planay (Savoia, Francia) (foto Manuela Mattone).

Per quanto attiene in particolare il patrimonio dell'idroelettricità, sono state di recente avviate una serie di attività volte a incrementare la sua fruizione da parte di un pubblico costituito non necessariamente da esperti e specialisti del settore, ma anche da famiglie, scolaresche, persone di diversa età ed estrazione culturale, interessate a comprendere il funzionamento dell'intero sistema produttivo e a capire dove e come viene prodotta quella energia di cui quotidianamente ciascuno di noi fruisce ogniqualvolta anche solo semplicemente accende la luce nella propria abitazione (Frantál, Urbánková, 2014). A tale scopo sono stati allestiti musei quali ad esempio il Museo dell'energia idroelettrica di Cedegolo, facente parte del sistema MUSIL (Museo dell'Industria e del Lavoro di Brescia7). Il museo di Cedegolo è ospitato all'interno di una ex-centrale idroelettrica dismessa nel 1962 e si propone di raccontare una fase importante dell'industrializzazione italiana, di valorizzare il patrimonio industriale e la cultura materiale della modernità, di diffondere la conoscenza di carattere scientifico e di favorire l'acquisizione di una maggiore consapevolezza relativamente ai temi dell'energia e dell'ambiante (Azzoni, 2008). L'itinerario espositivo è stato pensato con il precipuo scopo di esplicitare in modo chiaro il percorso seguito dall'acqua per consentire la produzione di energia elettrica, mettendo in evidenza il ruolo fondamentale che la ricerca scientifica e

\footnotetext{
${ }^{7}$ Il Musil è il primo complesso museale in Italia espressamente dedicato al tema dell'industrializzazione inteso come fenomeno complesso che ha coinvolto l'intera società nel corso degli ultimi secoli. Le esposizioni, allestite in diversi musei, si concentrano sulle tematiche dell'industria e del lavoro, intesi come principali forze motrici del processo di modernizzazione.
} 
lo sforzo compiuto dagli uomini (siano essi tecnici od operai) hanno avuto nel contribuire alla modernizzazione del Paese. Altrettanto interessante è la Galerie Hydraulica a Le Planay in Savoia (Francia), il cui allestimento consente di ripercorrere lo sviluppo industriale della vallata di Bozel (Savoia, Francia) caratterizzata dalla presenza di insediamenti produttivi alimentati dall'energia prodotta nelle centrali idroelettriche ivi realizzate e tuttora in funzione 8 .

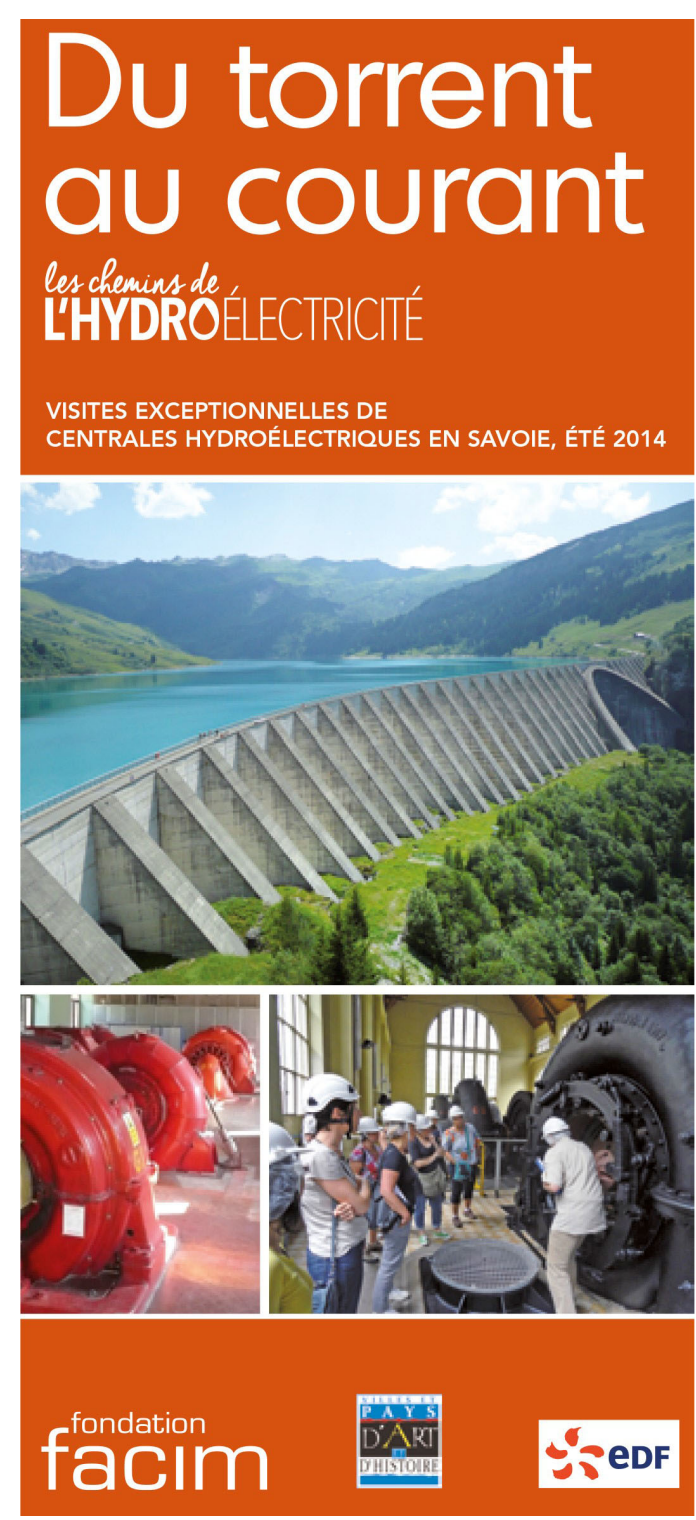

Figura 9. Brochure informativa dell'iniziativa Les chémins de l'hydroélectricité promossa da Fondazione Facim e EDF.
Accanto ai musei dedicati esistono poi una serie di altre attività che consentono di avvicinarsi al tema dell'idroelettricità e del suo patrimonio. Si tratta di visite guidate organizzate dalle stesse compagnie elettriche, talvolta in collaborazione con enti culturali. Estremamente interessanti sono in questo senso gli esempi offerti dalle attività organizzate nei territori della Savoia dalla Fondation Facim ${ }^{9}$ in collaborazione con EDF10, in Val d'Aosta dalla Compagnia Valdostana delle Acque ${ }^{11}$, in trentino dal Gruppo Dolimiti Energia12. In alcuni casi si tratta di visite associate a manifestazioni ed eventi allestiti nei luoghi di produzione dell'energia elettrica, in altri di mostre esperienziali che vedono l'attivo coinvolgimento dei visitatori oggi [...]

[...] sempre più interessati a vivere esperienze turistiche nuove creative, mentalmente, socialmente ed emotivamente coinvolgenti (Ferrari, Feltri, 2007, p. 1),

Tali attività hanno visto nel tempo aumentare e diversificarsi sia le proposte, sia il numero e la tipologia dei partecipanti 13 , a testimonianza del crescente interesse suscitato da queste iniziative il cui successo potrebbe incrementare qualora ampliasse l'offerta, coinvolgendo altre risorse presenti sul territorio, siano esse legate alla produzione di energia elettrica o da questa svincolate (Jirika et al., 2011).

\section{Limiti e proposte}

L'esame delle differenti proposte sopra descritte pone in evidenza come per ciascuna di esse l'attenzione venga principalmente focalizzata sul modo in cui viene prodotta l'energia elettrica mentre risulta ancora debole, o mancante, la diffusione di informazioni volte a valorizzare quelle tracce che, ancora oggi, testimoniano la storia dei manufatti architettonici e delle opere infrastrutturali sia dal punto di vista costruttivo, sia per ciò che attiene gli sforzi e l'impegno profusi

\footnotetext{
8 Per un maggiore approfondimento si veda www.galerie-hydraulica.com.

${ }^{9}$ La Fondation Facim si propone quale missione la diffusione della conoscenza e la valorizzazione del patrimonio e della cultura propri dei paesi della Savoia. Essa persegue tali obiettivi attraverso la messa in rete di duecento siti di rilievo mediante la messa a punto di itinerari tematici (tra cui Les chemins de l'hydroélectricité) volti a favorire la scoperta e la conoscenza del patrimonio culturale di detto territorio.

10 EDF è la maggiore azienda produttrice e distributrice di energia elettrica in Francia.

${ }^{11} \mathrm{Si}$ veda tal proposito quanto riportato sul sito http://www.cvaspa.it/mostre_eventi/home/index.jsp.

12 Per un maggiore approfondimento si veda il sito: http://www.hydrotourdolomiti.it/content/it/riva-del-garda

${ }^{13}$ Basti pensare che nel 2016 il numero delle che ha preso parte alla visita della centrale di Ponale a Riva del Garda, organizzata da Gruppo Dolomiti Energia, ha raggiunto quota 10.000.
} 
dagli uomini che hanno reso possibile la realizzazione di tali opere, di sacrifici e implicazioni che questi interventi hanno avuto sul paesaggio ${ }^{14}$ e sulle persone insediate in quei territori.

Le esposizioni museali, le visite alle centrali pongono l'accento sul percorso compiuto dall'acqua, sui modi e sugli strumenti (turbine, alternatori) che consentono la produzione dell'energia elettrica, dimenticando spesso il percorso fatto dagli uomini che tutto ciò hanno reso possibile. Oggi

i paesaggi elettrici scivolano nella memoria del paese; una memoria ricca e preziosa, che sarebbe uno spreco imperdonabile abbandonare all'oblio e alla dispersione (Caravaggi, 1998, p. 103).

Le tracce di questo percorso sono tuttora presenti sul territorio, talvolta nascoste, sovente in stato di rudere, e inglobate nella natura che si è progressivamente riappropriata dei propri spazi. Queste tracce costituiscono, a tutti gli effetti, testimonianze storico-culturali che, adeguatamente esplicitate e messe in rete, potrebbero divenire una risorsa capace di attrarre un turismo non necessariamente esperto, interessato ad approfondire le diverse tematiche connesse alla produzione di energia elettrica e a conoscere la storia di un Paese, di ciò che ha reso possibile il suo progresso e delle vallate montane le cui risorse idriche vengono sfruttate per scopi energetici. Queste stesse risorse potrebbero/dovrebbero essere messe a loro volta in rete con altre (siano esse naturalistiche, architettoniche, culturali o enogastronomiche) presenti negli stessi territori, contribuendo ad allargare il bacino di utenti potenzialmente interessati a fruire degli stessi. Ne potrebbe in tal modo scaturire la produzione di esternalità positive, indispensabili a favorire l'acquisizione delle risorse finanziarie grazie alle quali sarebbe possibile sia garantire la conservazione dei manufatti nel tempo, sia contribuire fattivamente alla rivitalizzazione dei territori montani.

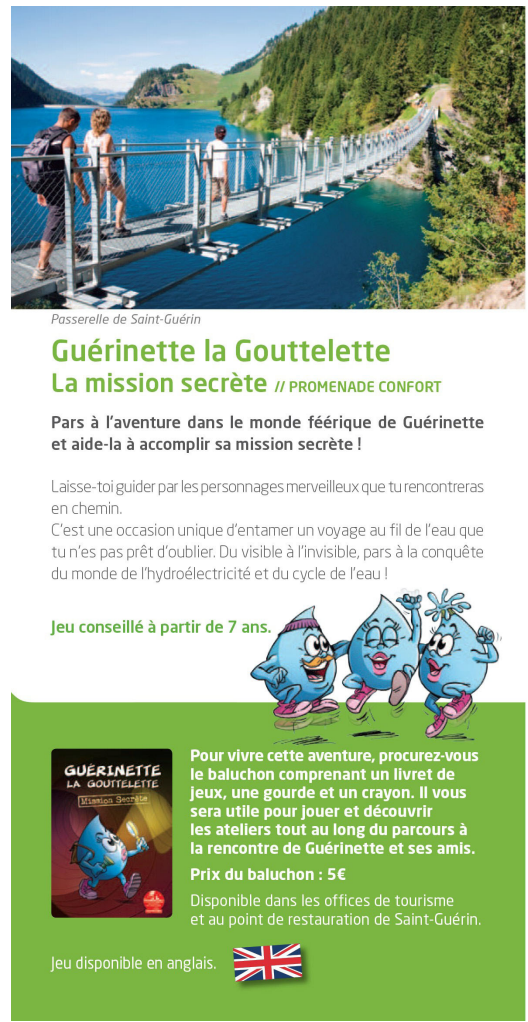

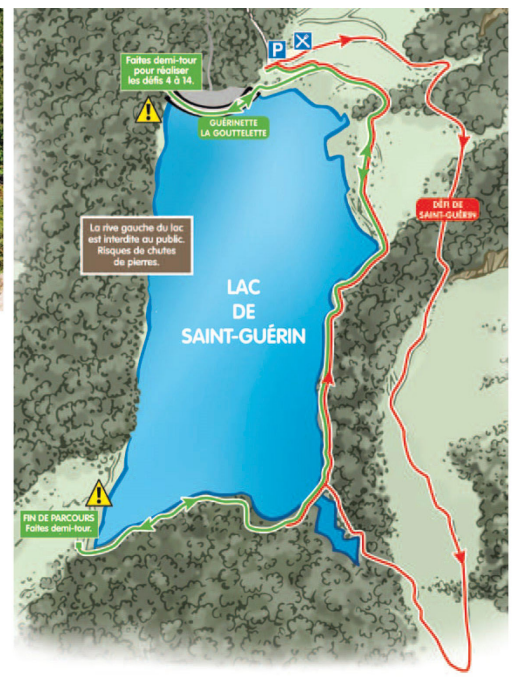

Deux parcours au choix deux univers pour découvrir I'hydroélectricité

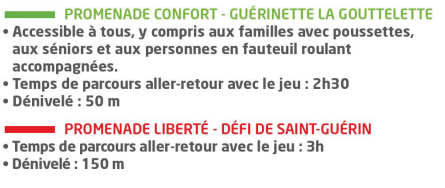

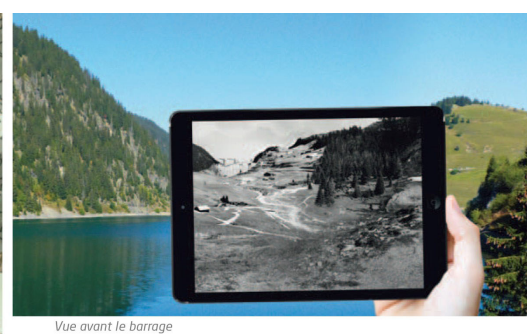

Défi de Saint-Guérin Construis le barrage ! / PROMENADE LIBERTÉ Remonte le temps jusqu'en 1957 et incarne l'ingénieur EDF en charge du chantier du barrage de Saint-Guérin. Tests en labo, études de terrain, rencontres des agriculteurs, tous les aspects de la construction d'un barpare tor Jeu conseillé à partir de 12 ans.

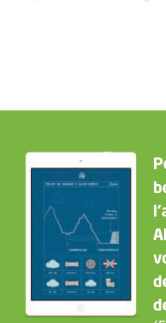
( ,

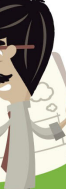
ur vivre cette aventure, vous a Iasoin dication "L'Empreinte des Grandes I'appilcation "L'Empreinte des Grandes
Alpes". SI vous ne l'avez pas téléchargée vous pouvez louer une tablette auprès des offices de tourisme ou au point
de restauration de Saint-Guérin de restauration de Saint-Guérin
(5E- caution et pièce didentité dem jeu disponible en anglais.

Figura 10. Brochure informativa delle Promenades savoyardes de découverte presso la diga di Saint Guérin.

\footnotetext{
${ }^{14}$ Paesaggio che è in taluni casi visibile, in altri no. Come sottolineato da Viviana Ferrario e Benedetta Castiglioni L'azione dei fattori naturali e ancor più la sedimentazione delle vicende storiche e delle pratiche che nel corso del tempo contribuiscono a modellare il paesaggio non sono [sempre] direttamente visibili: il paesaggio stesso permette di riconoscerle solo in piccola parte (Ferrario, Castiglioni, 2015, p. 533).
} 
La mutua collaborazione tra enti locali15 (Regione, ufficio del turismo, associazioni culturali) e le compagnie elettriche potrebbe consentire la messa a punto di percorsi che, legando l'escursionismo alla conoscenza dei sistemi idroelettrici (Pavia, 1998), offrano la possibilità di rintracciare i numerosi segni - oggi solo parzialmente visibili - dell'operato degli uomini che tali opere hanno realizzato e, al contempo, di fruire di queste e di tutte le altre risorse che caratterizzano tali contesti. Infine, dal momento che il paesaggio consente oggi di riconoscere e leggere solo in modo parziale tali segni e che

la dimensione visibile non potrà mai da sola restituire né i paesaggi del passato nelle loro fattezze, né il complesso intreccio delle dinamiche da cui queste stesse fattezze hanno tratto e traggono origine (Ferrario, Castiglioni, 2015, p. 533),

lo sfruttamento delle nuove opportunità offerte dai media digitali potrebbe contribuire in modo significativo alla lettura, alla comprensione e conseguentemente alla conservazione di questo patrimonio. Alcuni tentativi sono già stati effettuati in tale direzione: è il caso, ad esempio, delle Promenades savoyardes de découvertes che è possibile effettuare costeggiando il lago artificiale di Saint Guérin (Savoia, Francia) ${ }^{16}$. Mentre ai bambini viene offerta la possibilità di compiere un'esperienza ludico-educativa che consente loro di apprendere il funzionamento del sistema idroelettrico, per i visitatori più adulti è stata messa a punto un'applicazione gratuita denominata "L'Empreinte des Grandes Alpes" che, avvalendosi di realtà aumentata, unitamente a testimonianze audio e video, consente al turista di rivivere le fasi della costruzione della diga e di comprendere i profondi mutamenti che la sua realizzazione ha comportato. Comprendere i manufatti, raccontarne la loro storia, facilitarne la fruizione sicuramente contribuire fattivamente alla costituzione di una comunità che, interessata al proprio passato e al proprio futuro, sia propensa a sentirsi coinvolta nell'attività di tutela e di trasmissione alle generazioni a venire dei beni materiali e immateriali di cui anche il patrimonio dell'elettricità consta (Pracchi, 2017).

\section{Riferimenti}

Álvarez Areces, M. A. (2007). Presentación. In M. A. Álvarez Areces (ed.). Arquitecturas, Ingenierías y Culturas del Agua. Gijón: Cicees.

Álvarez Areces, M. A. (2017). Las rutas del kilowatio a través de la ingeniería, la arquitectura y el arte. In M. Mattone, \& E. Vigliocco. Patrimonio y Paisajes Eléctricos. Gijón: Cicees, pp. 115-144.

Archesso, B. (2016). La regina delle cascate torna all'antico splendore. In La Stampa, 3 giugno, p. 59.

Azzoni, G. (2015). Il museo dell'energia idroelettrica a Cedegolo. San Zeno Naviglio (Brescia): Grafo edizioni.

Bettini, G. (1991). "L'uomo, l'ambiente, le risorse del territorio valtellinese", in Fortezze gotiche e lune elettriche. Le centrali elettriche della AEM in Valtellina. Milano: Aem, pp. 57-83.

Boccioni, U. (1914). La pittura futurista. In Z. Birolli (ed.) (1971). Umberto Boccioni. Scritti editi e inediti. Milano: Feltrinelli.

Caravaggi, L. (1998). Natura ed energia. Conflitti e progetti di ricomposizione. In R. Pavia (ed.) (1998). Paesaggi elettrici. Territori architetture culture. Venezia: Marsilio, pp. 97-116.

Casanova, J. F. (2017), Comme un saphir dans son écrin de verdure. In le dauphiné. 14 agosto, p. 28.

Castellano, A. (1991). Archeologia industriale degli impianti idroelettrici in Valtellina. In Fortezze gotiche e lune elettriche. Le centrali elettriche della AEM in Valtellina. Milano: Aem, pp. 119-144.

${ }^{15} \mathrm{Si}$ veda a tal proposito quanto riportato in Jiricka, A., Salak, B., Wirth, V., Pröbstl-Haider, U. (2012), Creating a powerful niche product - ways to successful branding of "energy-tourism", Keller, P., Laessen, C. New Challenges for Tourism Promotion. Berlino: ESV, pp. 85-103.

${ }^{16} \mathrm{http}: / /$ areches-beaufort.com/noesit/!/fiche/l-empreinte-des-grandes-alpes-itineraire-saint-guerin-construction-dubarrage-664461.

(c) Labor \& Engenho, Campinas [SP] Brasil, v.11, n.4, p.426-435, out./dez. 2017. 
Choay, F. (1995). L'allegoria del patrimonio. Roma: Officina Edizioni.

Codice dei Beni Culturali e del Paesaggio, D. Lgs. 22 gennaio 2004, n. 42.

Di Battista, V. (2011). Archeologia industriale e paesaggio. Problemi di valorizzazione. In M. Ramello (ed.) (2011). La riconversione del patrimonio industriale. Il caso del territorio casalese nella prospettiva italiana ed europea. Firenze: Alinea, pp. 92-99.

Ferrari, S., \& Feltri, A. R. (2007). L'approccio esperienziale ai beni culturali come strumento di differenziazione dell'offerta turistica. Consultabile in http://www.fizz.it/home/sites/default /files/allegati/articoli/ pdf_articoli_completi/2007-ferrari_veltri.pdf

Ferrario, V., \& Castiglioni, B. (2015). Il paesaggio invisibile delle transizioni energetiche. Lo sfruttamento idroelettrico del bacino del Piave. In Bollettino della Società Geografica Italiana, vol. VIII, pp. 531-553.

Frantál, B., \& Urbánková, R. (2014). Energy tourism: An emerging field of study. In Current Issues in Tourism, December, pp. 1-18.

Jiricka, A., Salak, B., Wirth, V., \& Pröbstl-Haider, U. (2012), Creating a powerful niche product - ways to successful branding of "energy-tourism", Keller, P., Laessen, C. New Challenges for Tourism Promotion. Berlino: ESV, pp. 85-103.

Mattone, M. (2017). Paisaje de la electricidad y arquitectura (pp. 42-55). In M. Mattone, \& E. Vigliocco. Patrimonio y Paisajes Eléctricos. Gijón: Cicees.

Pavia, R. (ed.) (1998). Paesaggi elettrici. Territori architetture culture. Venezia: Marsilio.

Pracchi, V. N. (2017). La 'domanda' o il 'bisogno' di fruizione dei beni culturali. In Prescia, R. (ed.), RICerca/ REStauro. Valorizzazione e gestione delle informazioni. Roma: Edizioni Quasar, pp. 857-866.

Selvafolta, O. (1998). "La costruzione del paesaggio idroelettrico nelle regioni settentrionali”. In R. Pavia (ed.) (1998). Paesaggi elettrici. Territori architetture culture. Venezia: Marsilio, pp. 41-71.

Toso, F. C. (2014). "A hydroelectric landscape in Italian Alps: elements, meanings, and design cues in a historical hydroelectric development in Alta Valtellina". Journal of Landscape Architecture, n. 2, pp. 30-39.

Vecco, M. (2007). L'evoluzione del concetto di bene culturale. Milano: FrancoAngeli

Vecco, M. (2010). A definition of cultural heritage: from the tangible to the untangible. Journal of Cultural Heritage, 11:321-324.

Vismara, A. (1925). Gli impianti idroelettrici in Italia ed il loro contributo all'economia del paese. La regolazione della idrologia di un paese a mezzo degli impianti elettrici. citato in Selvafolta, 0. (1998). "La costruzione del paesaggio idroelettrico nelle regioni settentrionali. In R. Pavia (ed.) (1998). Paesaggi elettrici. Territori architetture culture. Venezia: Marsilio, pp. 41-71.

\subsection{Sitografia}

http://fondation-facim.fr/fr/le-pays-dart-et-dhistoire/les-thematiques/les-chemins-de-lhydroelectricite (consultato in data 30 aprile 2017)

http://www.cvaspa.it/giri_energia/giri_energia/ (consultato in data 30 aprile 2017)

http://www.hydrotourdolomiti.it/content/it/home (consultato in data 7 giugno 2017)

http://areches-beaufort.com/noesit/!/fiche/l-empreinte-des-grandes-alpes-itineraire-saint-guerinconstruction-du-barrage-664461 (consultato in data 16 agosto 2017).

www.galerie-hydraulica.com (consultato in data 16 agosto 2017).

(C) Labor \& Engenho, Campinas [SP] Brasil, v.11, n.4, p.426-435, out./dez. 2017. 